\title{
Fock representations of ZF algebras and $R$-matrices
}

\author{
Gandalf Lechner $^{1}$. Charley Scotford ${ }^{1}[$ (]) \\ Received: 22 October 2019 / Revised: 20 January 2020 / Accepted: 12 February 2020 / \\ Published online: 5 March 2020 \\ (c) The Author(s) 2020
}

\begin{abstract}
A variation of the Zamolodchikov-Faddeev algebra over a finite-dimensional Hilbert space $\mathcal{H}$ and an involutive unitary $R$-Matrix $S$ is studied. This algebra carries a natural vacuum state, and the corresponding Fock representation spaces $\mathcal{F}_{S}(\mathcal{H})$ are shown to satisfy $\mathcal{F}_{S \boxplus R}(\mathcal{H} \oplus \mathcal{K}) \cong \mathcal{F}_{S}(\mathcal{H}) \otimes \mathcal{F}_{R}(\mathcal{K})$, where $S \boxplus R$ is the box-sum of $S$ (on $\mathcal{H} \otimes \mathcal{H}$ ) and $R$ (on $\mathcal{K} \otimes \mathcal{K}$ ). This analysis generalises the well-known structure of Bose/Fermi Fock spaces and a recent result of Pennig. These representations are motivated from quantum field theory (short-distance scaling limits of integrable models).
\end{abstract}

Keywords Yang-Baxter · Representation theory - GNS construction · Fock space · R-Matrices · Zamolodchikov-Faddeev algebra

Mathematics Subject Classification 16T25 · 16G99 · 30H20

\section{Introduction}

The Zamolodchikov-Faddeev algebras were introduced by the Zamolodchikov and Faddeev as a tool to describe integrable quantum field theories in which the full $S$ matrix factorises into products of 2-particle $S$-matrices [8,25]. These algebras (called ZF algebras for short) are generated by "creation" and "annihilation" operators obeying quadratic exchange relations given by the 2-particle $S$-matrix. ZF algebras generalise the familiar CCR and CAR algebras [3] and are closely related to Wick algebras that allow normal ordering [11]. Hilbert space representations of ZF algebras are of central importance in integrable quantum field theory (see, for example, $[1,5,14,18,24]$ for a few sample publications from a very large body of literature) as well as in other fields such as $q$-deformations [20,22] or anyonic statistics [15].

\footnotetext{
Charley Scotford

ScotfordC@Cardiff.ac.uk

Gandalf Lechner

LechnerG@Cardiff.ac.uk

1 School of Mathematics, Cardiff University, Cardiff CF24 4AG, UK
} 
The central datum defining the relations in a $\mathrm{ZF}$ algebra is a solution of the YangBaxter equation. In the QFT context, this solution plays the role of elastic two-particle scattering matrix and depends on a spectral parameter (rapidity), i.e. it is a matrixvalued function $\mathbb{R} \ni \theta \mapsto \mathbf{S}(\theta) \in \operatorname{End}(\mathcal{H} \otimes \mathcal{H})$, where $\mathcal{H}$ is a finite-dimensional Hilbert space.

The present work has its background in an ongoing research project about shortdistance scaling limits of integrable quantum field theories on two-dimensional Minkowski space [23]. At finite scale, the QFTs that we have in mind are specified in an inverse scattering approach in terms of a mass parameter $m>0$ and a Fock (vacuum) representation of the ZF algebra given by a two-particle $S$ matrix $\mathbb{R} \ni \theta \mapsto \mathbf{S}(\theta) \in \operatorname{End}(\mathcal{H} \otimes \mathcal{H})$, subject to the usual constraints of unitarity, Poincaré invariance, Hermitian analyticity, and crossing symmetry. Under suitable regularity assumptions, a quantum field theory which has $\mathbf{S}$ as its two-particle $S$-matrix exists and can be constructed in the framework of algebraic quantum field theory [10] from $m$ and $\mathbf{S}$ alone, without reference to a Lagrangian (see [14] for a review of this construction).

It is then an interesting question to study the conformal chiral short-distance scaling limit of these theories, generalising previous work on scalar (meaning $\mathcal{H}=\mathbb{C}$ ) models [2]. In our inverse scattering approach, field operators that are localised in Rindler wedges can be constructed explicitly in terms of the Fock representation of the ZF algebra, but the physical fields are characterised only indirectly. Hence, it is not clear for which $\mathbf{S}$ the scaling limit exists as a local QFT or what its properties are (although this is investigated in many specific models [9]).

It is known, however, that existence of the two limits $S_{ \pm}:=\lim _{\theta \rightarrow \pm \infty} \mathbf{S}(\theta)$ is a necessary condition for the existence of the scaling limit, and local chiral fields have to satisfy specific commutation relations with representations (of the symmetric or braid groups) derived from $S_{ \pm}$. Furthermore, the value $S_{0}:=\mathbf{S}(0)$ of the $S$-matrix at zero rapidity transfer determines the (anti-)commutation relations between the chiral theories on the two opposite lightrays. Thus the three $\theta$-independent unitary solutions $S_{0}, S_{+}, S_{-}$of the Yang-Baxter equation (we will refer to unitary solutions to the Yang-Baxter equation as "R-matrices" in this article) derived from $\mathbf{S}$ govern essential properties of the scaling limit theories.

This motivates a closer study of representations of ZF algebras built from $R$ matrices, which is the subject of this article. The properties of two-particle $S$-matrices imply that $S_{0}$ is involutive (meaning $S_{0}^{2}=1$ ) and $S_{+}^{*}=S_{-}$. In many examples (such as diagonal $S$-matrices or the $S$-matrix of the $O(N)$ sigma models), the limits $S_{ \pm}$ are involutive as well. Although our arguments in this article are completely independent of QFT models, we take this physical background into account and mostly consider ZF algebras built from involutive $R$-matrices. This enables us to use and apply recently established tools and results about the mathematical structure of the space of all involutive $R$-matrices [16], denoted $\mathcal{R}_{0}(\mathcal{H})$ for base space $\mathcal{H}$.

In Sect. 2, we define $\mathrm{ZF}$ algebras as abstract unital *-algebras and their natural normalised vacuum functional $\omega$. Section 3 is then devoted to an analysis of Fock representations. If $\omega$ is positive as a functional (hence a state), these Fock representations coincide with the representations given by the general Gelfand-Naimark-Segal (GNS) construction applied to the ZF algebra w.r.t. $\omega$. However, in general it is not 
initially clear that $\omega$ is positive, and an independent construction has to be given. We do this by adapting a concrete representation known from quantum field theory to our setting and verify the GNS property afterwards (Theorem 3.4). Given the ZF algebra based on an involutive $R$-matrix $S \in \mathcal{R}_{0}(\mathcal{H})$, this provides us with an $S$-symmetric Fock representation space $\mathcal{F}_{S}(\mathcal{H})$. For special choices of $S$, this coincides with the Bose or Fermi Fock space over $\mathcal{H}$.

In Sects. 4 and 5, we study the dependence of $\mathcal{F}_{S}(\mathcal{H})$ on $S$. Adopting the boxsum operation $\boxplus$ on $\bigcup_{\mathcal{H}} \mathcal{R}_{0}(\mathcal{H})$ from [16] (which yields $R \boxplus S \in \mathcal{R}_{0}(\mathcal{H} \oplus \mathcal{K}$ ) for $\left.R \in \mathcal{R}_{0}(\mathcal{H}), S \in \mathcal{R}_{0}(\mathcal{K})\right)$, we prove

$$
\mathcal{F}_{R \boxplus S}(\mathcal{H} \oplus \mathcal{K}) \cong \mathcal{F}_{R}(\mathcal{H}) \otimes \mathcal{F}_{S}(\mathcal{K})
$$

in Theorem 5.3 as our main result. It is interesting to note that for the case that all resulting Fock spaces are finite-dimensional (which requires $R, S$ to have "Fermionic" behaviour), such an isomorphism was recently proven by Pennig [21] with quite different methods as part of his classification of polynomial exponential functors on the category of finite-dimensional Hilbert spaces. As required for applications in quantum field theory, our result holds for infinite-dimensional Fock spaces and does not only give an isomorphism of Hilbert spaces, but also an isomorphism of representations of ZF algebras.

Isomorphism (1.1) is of particular interest because up to a natural notion of equivalence, every involutive $R$-matrix can be written as an iterated box-sum of finitely many very simple $( \pm 1) R$-matrices [16]. In the QFT context, these correspond to free field theories, and the resulting decomposition of the Fock spaces and representations of $\mathrm{ZF}$ algebras could turn out to be a tool to investigate whether the scaling limit is isomorphic to a free field theory. As a first step in this direction, we investigate in Sect. 5 under which conditions we may identify our Fock representation with tensor products of simpler ones, providing examples and counterexamples.

Applications of our findings to the mentioned quantum field theoretic models and an analysis of their short-distance scaling limits will appear in a future work.

\section{An abstract ZF algebra}

We begin by abstractly defining a variation of the well-known ZamolodchikovFaddeev algebra [8,25]. Let $\mathcal{L}$ be a (separable) Hilbert space and $S$ be a set of $d^{4}(d \in \mathbb{N})$ complex numbers whose elements are labelled by symbols $S_{\delta \gamma}^{\alpha \beta}$ where $\alpha, \beta, \delta, \gamma \in\{1, \ldots, d\}$. We then define the unital $*$-algebra $\mathcal{Z}(S, \mathcal{L})$ as the algebra generated by the symbols $1_{\mathcal{Z}(S, \mathcal{L})}, Z_{1}(f), Z_{2}(f), \ldots, Z_{d}(f)$ for all $f \in \mathcal{L}$ which obey the following exchange relations:

$$
\begin{aligned}
& Z_{\alpha}(f) Z_{\beta}(g)=S_{\delta \gamma}^{\beta \alpha} Z_{\gamma}(g) Z_{\delta}(f), \\
& Z_{\alpha}(f) Z_{\beta}^{*}(g)=S_{\beta \delta}^{\alpha \gamma} Z_{\gamma}^{*}(g) Z_{\delta}(f)+\delta_{\beta}^{\alpha} \cdot\langle f, g\rangle_{\mathcal{L}} 1_{\mathcal{Z}(S, \mathcal{L})},
\end{aligned}
$$


where we understand that the repeated indices in an expression imply the sum over all possible values (Einstein summation convention).

We may view $S$ as a linear map over the tensor square of a Hilbert space $\mathcal{H}$ of dimension $d$. Then the numbers $S_{\delta \gamma}^{\alpha \beta}$ can be viewed as the matrix elements $\left\langle e_{\alpha} \otimes\right.$ $\left.e_{\beta}, S\left(e_{\delta} \otimes e_{\gamma}\right)\right\rangle$, where $\left(e_{\alpha}\right)_{\alpha=1}^{d_{\mathcal{H}}}$ is an orthonormal basis of $\mathcal{H}$.

A class of algebras related to ZF algebras, the so-called Wick algebras, are defined by omitting (2.2) from the definition. Their representations have been studied in $[6,11,12]$.

At this stage it is not at all clear whether or not there exist Hilbert space representations of $\mathcal{Z}(S, \mathcal{L})$ - for example, in [11, p. 18] it is shown that for certain choices of $S$ the corresponding Wick algebra admits no Hilbert space representations. As we will see, under certain assumptions on $S$ a GNS representation of $\mathcal{Z}(S, \mathcal{L})$ can be constructed.

The notion of Wick ordering (sometimes also known as "normal" ordering) is a prolific and useful concept in the analysis of these algebras. To write an element $X \in \mathcal{Z}(S, \mathcal{L})$ in Wick ordered form means to apply the governing relation (2.2) such that $X$ becomes of the form

$$
\sum_{\eta, \xi} \zeta_{\eta, \xi} Z_{\eta}^{*}\left(f_{\eta}\right) Z_{\xi}\left(g_{\xi}\right)
$$

where $\zeta_{\eta, \xi} \in \mathbb{C}$. The multi-index notation we have adopted here can be read as, for example,

$$
Z_{\eta}^{*}\left(f_{\eta}\right)=Z_{\eta_{1}}^{*}\left(f_{\eta_{1}}\right) Z_{\eta_{2}}^{*}\left(f_{\eta_{2}}\right) \cdots Z_{\eta_{N}}^{*}\left(f_{\eta_{N}}\right)
$$

where all $f_{\eta_{n}} \in \mathcal{L}$ and $|\boldsymbol{\eta}|=N \in \mathbb{N}_{0}$ and in case $|\boldsymbol{\eta}|=0$, we take $Z_{\eta}^{*}\left(f_{\eta}\right)=1_{\mathcal{Z}(S, \mathcal{L})}$. We also remark that for the case of $|\boldsymbol{\eta}|=|\xi|=0$ we have just a multiple of the identity in (2.3). Every element of $\mathcal{Z}(S, \mathcal{L})$ can be written in Wick ordered form. The Wick ordered form is typically not unique as we may exchange any two $Z$ or $Z^{*}$ elements in the expression using (2.1). However, the term with $|\boldsymbol{\eta}|=|\boldsymbol{\xi}|=0$ is unique.

This discussion facilitates the definition of a linear functional over $\mathcal{Z}(S, \mathcal{L})$ and in particular proves it to be uniquely defined by the properties specified in the following definition.

Definition 2.1 We define a normalised linear functional $\omega: \mathcal{Z}(S, \mathcal{L}) \rightarrow \mathbb{C}$ by the properties

$$
\omega\left(1_{\mathcal{Z}(S, \mathcal{L})}\right)=1
$$

(ii)

$$
\omega\left(Z_{\alpha}^{*}(f) \cdot X\right)=0,
$$


(iii)

$$
\omega\left(X \cdot Z_{\alpha}(f)\right)=0
$$

for all $\alpha \in\{1, \ldots, d\}, f \in \mathcal{L}$ any $X \in \mathcal{Z}(S, \mathcal{L})$.

Defining a second functional as $\lambda(X):=\overline{\omega\left(X^{*}\right)}$ and applying uniqueness, we see that $\omega$ is Hermitian, but it is not necessarily positive.

Example 2.2 We consider here some simple examples of $\mathcal{Z}(S, \mathcal{L})$.

If we take first $S_{\delta \gamma}^{\beta \alpha}= \pm \delta_{\delta}^{\alpha} \delta_{\gamma}^{\beta}$, where $\delta$ is the Kronecker delta, relations (2.1) and (2.2) now read (for $f, g \in \mathcal{L}$ )

$$
\begin{aligned}
& Z_{\alpha}(f) Z_{\beta}(g)= \pm Z_{\beta}(g) Z_{\alpha}(f), \\
& Z_{\alpha}(f) Z_{\beta}^{*}(g)= \pm Z_{\beta}^{*}(g) Z_{\alpha}(f)+\delta_{\beta}^{\alpha} \cdot\langle f, g\rangle_{\mathcal{L}}
\end{aligned}
$$

Choosing an orthonormal basis $\left(e_{\alpha}\right)_{\alpha=1}^{d}$ of $\mathbb{C}^{d}$, one realises that the elements $a(\cdot)$ defined as $a\left(e_{\alpha} \otimes f\right):=Z_{\alpha}(f)$ satisfy the governing relations of the $\operatorname{CCR}\left(\mathbb{C}^{d} \otimes \mathcal{L}\right)$ $(+)$ and $\operatorname{CAR}\left(\mathbb{C}^{d} \otimes \mathcal{L}\right)(-)$ algebras [3,7], respectively. We will note more on their representations in the next section. If we instead take $S_{\delta \gamma}^{\alpha \beta}=-\delta_{\delta}^{\alpha} \delta_{\gamma}^{\beta}$, the governing relations become

$$
\begin{gathered}
Z_{\alpha}(f) Z_{\beta}(g)=-Z_{\alpha}(g) Z_{\beta}(f) \\
Z_{\alpha}(f) Z_{\beta}^{*}(g)=\delta_{\beta}^{\alpha}\left(-\sum_{\delta} Z_{\delta}^{*}(g) Z_{\delta}(f)+1_{\mathcal{Z}(1, \mathcal{L})}\right) .
\end{gathered}
$$

This example is interesting in the context of Fock representations (which we will discuss in the next section), but for now we simply note that this example is also explored for the case of $\mathcal{L}=\mathbb{C}$ in $[11$, p. 48] in a Wick algebraic setting as a "degenerate case".

\section{Fock representations}

We now consider (pre-)Hilbert space representations of $\mathcal{Z}(S, \mathcal{L})$. To this end, we take the tensor product $\tilde{\mathcal{H}}:=\mathcal{H} \otimes \mathcal{L}$ (we reserve the notation of the tilde signifying the tensor product with $\mathcal{L}$ ) of a Hilbert space $\mathcal{H}$ (of finite dimension $d_{\mathcal{H}}$ ) and the second internal space $\mathcal{L}$ for which we do not specify (or require) finite dimensionality. As mentioned previously, we may fix an orthonormal basis $\left(e_{\alpha}\right)_{\alpha=1}^{d_{\mathcal{H}}}$ of $\mathcal{H}$ and view the parameters $S_{\delta \gamma}^{\alpha \beta}$ in $(2.1,2.2)$ as the matrix elements $S_{\delta \gamma}^{\alpha \beta}=\left\langle e_{\alpha} \otimes e_{\beta}, S\left(e_{\delta} \otimes e_{\gamma}\right)\right\rangle$ of a linear map $S: \mathcal{H} \otimes \mathcal{H} \rightarrow \mathcal{H} \otimes \mathcal{H}$.

As discussed in Introduction, construction in quantum field theory motivates us to consider the case that $S$ solves the Yang-Baxter equation and is involutive. Note that $S$ solving the Yang-Baxter equation implies that $\mathcal{Z}(S, \mathcal{L})$ is associative. 
Definition 3.1 Let $\mathcal{H}$ be a Hilbert space. An involutive $R$-matrix on $\mathcal{H}$ is a unitary, involutive map $S \in \mathcal{B}(\mathcal{H} \otimes \mathcal{H})$ that solves the Yang-Baxter equation. That is

$$
\begin{gathered}
S=S^{*}=S^{-1}, \\
\left(S \otimes 1_{\mathcal{H}}\right)\left(1_{\mathcal{H}} \otimes S\right)\left(S \otimes 1_{\mathcal{H}}\right)=\left(1_{\mathcal{H}} \otimes S\right)\left(S \otimes 1_{\mathcal{H}}\right)\left(1_{\mathcal{H}} \otimes S\right) .
\end{gathered}
$$

We also denote by $\mathcal{R}_{0}(\mathcal{H})$ the set of all involutive $R$-matrices on $\mathcal{H}$.

We will mostly be interested in the case where $\mathcal{H}$ is finite-dimensional (which we will always explicitly state), otherwise we always take $\mathcal{H}$ to be separable.

Before beginning the discussion of representations of $\mathcal{Z}(S, \mathcal{L})$, it is necessary to extend the definition of $S$ to involve the space $\mathcal{L}$ and to do so we introduce the unitary operator $U_{n}:(\mathcal{H} \otimes \mathcal{L})^{\otimes n} \rightarrow \mathcal{H}^{\otimes n} \otimes \mathcal{L}^{\otimes n}$ defined by

$$
U_{n}\left(\bigotimes_{i=1}^{n}\left(h_{i} \otimes f_{i}\right)\right)=\left(\bigotimes_{i=1}^{n} h_{i}\right) \otimes\left(\bigotimes_{i=1}^{n} f_{i}\right), \quad h_{i} \in \mathcal{H}, f_{i} \in \mathcal{L} .
$$

We can easily see that $U_{n}$ is unitary from the above expression and it can be thought of as "disentangling" contributions from both Hilbert spaces, which induces an isomorphism between the domain and codomain of $U_{n}$; hence, we will explicitly describe data acting only on $\mathcal{H}^{\otimes n} \otimes \mathcal{L}^{\otimes n}$ in this section. Employing the bounded linear operator $\mathcal{B}(\mathcal{L} \otimes \mathcal{L}) \ni F:=F_{\mathcal{L}}$ (the tensor flip), we write

$$
S_{F}:=S \otimes F: \mathcal{H}^{\otimes 2} \otimes \mathcal{L}^{\otimes 2} \rightarrow \mathcal{H}^{\otimes 2} \otimes \mathcal{L}^{\otimes 2},
$$

which one readily checks is still unitary, involutive and a solution to the Yang-Baxter equation. Though this is the explicit operator used in the construction, the interest is mostly in the contributions from $S$, and so we will avoid using $S_{F}$ in further notation where possible.

We would like to consider the GNS representation of $\mathcal{Z}(S, \mathcal{L})$ with respect to the functional $\omega$, but at this stage it is unclear whether $\omega$ is positive. Instead, we will independently construct a representation of $\mathcal{Z}(S, \mathcal{L})$ and show it has the GNS property.

We choose an orthonormal basis $\left(e_{\alpha}\right)_{\alpha=1}^{d_{\mathcal{H}}}$ of $\mathcal{H}$ and also make use of multi-index notation where we take $e_{\alpha} \in \mathcal{H}^{\otimes n}$ to mean $e_{\alpha_{1}} \otimes \cdots \otimes e_{\alpha_{n}}$.

Let $S \in \mathcal{R}_{0}(\mathcal{H})$, then we recall the structure of a Hilbert space representation of $\mathcal{Z}(S, \mathcal{L})$ as laid in a field theoretic setting in [17]. Denote by $S_{k, n}:=1^{\otimes(k-1)} \otimes S \otimes$ $1^{\otimes(n-k-1)}$ (where $\left.S\left(e_{\alpha} \otimes e_{\beta}\right)=S_{\alpha \beta}^{\gamma \delta} e_{\gamma} \otimes e_{\delta}\right)$ then we construct unitary operators on $\mathcal{H}^{\otimes n} \otimes \mathcal{L}^{\otimes n}:$

$$
D_{n}^{S}\left(\tau_{i}\right)=S_{i, n} \otimes F_{i}
$$

where $\tau_{i} \in \mathfrak{S}_{n}$ (the symmetric group of $n$ letters) is a transposition, swapping nearest neighbour $i$ and $(i+1)$ th elements. It is straightforward to see that these operators generate a unitary representation of $\mathfrak{S}_{n}$ on $\mathcal{H}^{\otimes n} \otimes \mathcal{L}^{\otimes n}$, and then we can define an orthogonal projection [17] by taking their mean: 


$$
P_{n}^{S}:=\frac{1}{n !} \sum_{\pi \in \mathfrak{S}_{n}} D_{n}^{S}(\pi) \in \mathcal{B}\left(\mathcal{H}^{\otimes n} \otimes \mathcal{L}^{\otimes n}\right) .
$$

Define now the spaces

$$
\tilde{\mathcal{H}}_{n}:=U_{n}^{*} P_{n}^{S} U_{n} \tilde{\mathcal{H}}^{\otimes n}
$$

then the $S$-symmetrised Fock space is given by

$$
\mathcal{F}_{S}(\tilde{\mathcal{H}}):=\bigoplus_{n \geq 0} \tilde{\mathcal{H}}_{n}
$$

For (3.3) to be a unitary representation of $\mathfrak{S}_{n}$, involutivity of $S$ is a crucial property. Dropping involutivity, unitary $R$-matrices only give representations of the Braid groups. The concept of an $S$-symmetric Fock space can be generalised to noninvolutive $S$ [19], but it won't play a role in the current work.

On this space, we have a vacuum vector $\Omega_{S}=1 \oplus 0 \oplus \ldots$, and a dense subspace $\mathcal{F}_{S}^{0}(\tilde{\mathcal{H}})$ (consisting of vectors of "finite particle" number, meaning they are terminating direct sums of elements in increasing tensor powers). There is a natural unitary $U$ from $\mathcal{F}_{S}(\tilde{\mathcal{H}})$ to the "disentangled Fock space" $\bigoplus_{n} P_{n}^{S}\left(\mathcal{H}^{\otimes n} \otimes \mathcal{L}^{\otimes n}\right)=: \bigoplus_{n} \mathcal{D}_{n}(\tilde{\mathcal{H}})$, namely $U=\bigoplus_{n} U_{n}$ is the second quantisation of the unitaries $U_{n}$ (3.2). We may therefore treat operators on $\mathcal{F}_{S}(\tilde{\mathcal{H}})$ and $U \mathcal{F}_{S}(\tilde{\mathcal{H}})$ on the same footing. To discuss the Fock representation of $\mathcal{Z}(S, \mathcal{L})$, it is more convenient to work on the latter space, and we define

$$
\begin{aligned}
z_{S}^{*}\left(e_{\xi} \otimes g\right) v_{n} \otimes f_{n} & :=\sqrt{n+1} P_{n+1}^{S}\left(e_{\xi} \otimes v_{n} \otimes g \otimes f_{n}\right), \\
z_{S}\left(e_{\xi} \otimes g\right) & :=\left(z_{S}^{*}\left(e_{\xi} \otimes g\right)\right)^{*},
\end{aligned}
$$

for $v_{n} \in \mathcal{H}^{\otimes n}, f_{n} \in \mathcal{L}^{\otimes n}, g \in \mathcal{L}$.

Remark 3.2 We can write the explicit action of $z_{S}$ in terms of the scalar product on $\tilde{\mathcal{H}}_{n}$ by

$$
\begin{aligned}
\left\langle w_{n-1} \otimes h_{n-1}, z_{S}\left(e_{\xi} \otimes g\right) v_{n} \otimes f_{n}\right\rangle & =\sqrt{n}\left\langle e_{\xi} \otimes w_{n-1} \otimes g \otimes h_{n-1}, v_{n} \otimes f_{n}\right\rangle, \\
z_{S}\left(e_{\xi} \otimes g\right) \Omega_{S} & =0,
\end{aligned}
$$

for $w_{n-1} \otimes h_{n-1} \in \mathcal{H}^{\otimes(n-1)} \otimes \mathcal{L}^{\otimes(n-1)}$. These operators then restrict to the symmetrised spaces $\mathcal{D}_{n}(\tilde{\mathcal{H}})$. In bra-ket notation, (3.5a) simply reads

$$
z_{S}\left(e_{\xi} \otimes g\right) v_{n} \otimes f_{n}=\sqrt{n}\left\langle e_{\xi} \otimes g\right| v_{n} \otimes f_{n} .
$$

We have defined $z_{S}^{*}, z_{S}$ with basis vectors of $\mathcal{H}$ as arguments; however, we can extend the definition to operators $z_{S}^{*}(\psi), \psi \in \tilde{\mathcal{H}}$ by linearity in their arguments (care to be taken when doing the same to $z_{S}$ as it is anti-linear in its argument).

For ease of notation, we will use the shorthand $z_{S}\left(e_{\alpha} \otimes g\right)=z_{S, \alpha}(g)$, and the polynomial algebra generated by all $z_{S, \alpha}(f), z_{S, \beta}^{*}(g), 1_{\tilde{\mathcal{H}}}$ we will denote by $\mathcal{P}_{S}$. 
Proposition 3.3 The vacuum vector $\Omega_{S}$ is cyclic for the algebra $\mathcal{P}_{S}$, that is, $\mathcal{P}_{S} \Omega_{S} \subset$ $U \mathcal{F}_{S}(\tilde{\mathcal{H}})$ is dense.

Proof Let $\psi \in \mathcal{F}_{S}(\tilde{\mathcal{H}})$ such that $\psi$ is orthogonal to $\mathcal{P}_{S} \Omega$. For any $n \in \mathbb{N}_{0}$, and vectors $f_{1}, \ldots, f_{n} \in \mathcal{L}, \alpha_{1}, \ldots, \alpha_{n} \in\left\{1, \ldots, d_{\mathcal{H}}\right\}$ we then have

$$
\begin{aligned}
0 & =\left\langle\psi, z_{S, \alpha_{1}}^{*}\left(f_{1}\right) \cdots z_{S, \alpha_{n}}^{*}\left(f_{n}\right) \Omega_{S}\right\rangle \\
& =\sqrt{n !}\left\langle\psi, P_{n}^{S}\left(e_{\alpha_{1}} \otimes \cdots \otimes e_{\alpha_{n}} \otimes f_{1} \otimes \cdots \otimes f_{n}\right)\right\rangle \\
& =\sqrt{n !}\left\langle\psi, e_{\alpha_{1}} \otimes \cdots \otimes e_{\alpha_{n}} \otimes f_{1} \otimes \cdots \otimes f_{n}\right\rangle
\end{aligned}
$$

where we have used that the projection $P_{n}^{S}$ is self-adjoint and leaves the symmetrised vector $\psi$ invariant. Since vectors of the form $e_{\alpha_{1}} \otimes \cdots \otimes e_{\alpha_{n}} \otimes f_{1} \otimes \cdots \otimes f_{n}$ form a total set in $\mathcal{H}^{\otimes n} \otimes \mathcal{L}^{\otimes n}$, we conclude that $\psi=0$. Thus $\Omega_{S}$ is cyclic for $\mathcal{P}_{S}$.

Before moving to the next result, we note specific elements of $\mathfrak{S}_{n}$ as they will play an important role in the following proof-define $\sigma_{n}:=\tau_{n-1} \tau_{n-2} \ldots \tau_{1} \in \mathfrak{S}_{n}$ which acts by taking the first element and moving it to the $n$-th position.

Theorem 3.4 Let $\mathcal{H}$ be a finite-dimensional Hilbert space and $S \in \mathcal{R}_{0}(\mathcal{H})$. Then the map $\pi_{S}: \mathcal{Z}(S, \mathcal{L}) \rightarrow \mathcal{P}_{S}$ given by

$$
\pi_{S}\left(1_{\mathcal{Z}(S, \mathcal{L})}\right):=1_{\tilde{\mathcal{H}}}, \quad \pi_{S}\left(Z_{\alpha}(f)\right):=z_{S, \alpha}(f)
$$

extends to a unital $*$-representation of $\mathcal{Z}(S, \mathcal{L})$ on $\mathcal{F}_{S}^{0}(\tilde{\mathcal{H}})$ with cyclic vector $\Omega_{S}$ and

$$
\omega(X)=\left\langle\Omega_{S}, \pi_{S}(X) \Omega_{S}\right\rangle . \quad(X \in \mathcal{Z}(S, \mathcal{L}))
$$

Proof We show first that the operators $z_{S, \alpha}(f), z_{S, \alpha}^{*}(f)$ satisfy (2.1) and (2.2) for all $f \in \mathcal{L}, \alpha \in\left\{1, \ldots, d_{\mathcal{H}}\right\}$. Let $v_{n} \otimes h_{n} \in \mathcal{D}_{n}(\tilde{\mathcal{H}}), f, g \in \mathcal{L}$ then taking into account $P_{n+2}^{S}=P_{n+2}^{S} S_{1, n+1} \otimes F_{1}$

$$
\begin{aligned}
z_{S, \alpha}^{*}(f) z_{S, \beta}^{*}(g) v_{n} \otimes h_{n} & =\sqrt{n+1} \sqrt{n+2} P_{n+2}^{S}\left(e_{\alpha} \otimes e_{\beta} \otimes v_{n} \otimes f \otimes g \otimes h_{n}\right) \\
& =\sqrt{n+1} \sqrt{n+2} P_{n+2}^{S}\left(S_{\alpha \beta}^{\gamma \delta} e_{\gamma} \otimes e_{\delta} \otimes v_{n} \otimes g \otimes f \otimes h_{n}\right) \\
& =S_{\alpha \beta}^{\gamma \delta} z_{S, \gamma}^{*}(g) z_{S, \delta}^{*}(f) v_{n} \otimes h_{n} .
\end{aligned}
$$

Given that $n$ and $v_{n} \otimes h_{n}$ were arbitrary, we read off

$$
z_{S, \alpha}^{*}(f) z_{S, \beta}^{*}(g)=S_{\alpha \beta}^{\gamma \delta} z_{S, \gamma}^{*}(g) z_{S, \delta}^{*}(f)
$$

as operators on $\mathcal{F}_{S}^{0}(\tilde{\mathcal{H}})$. Taking adjoints of both sides and applying both the unitarity and involutivity of $S$, we arrive at equation (2.1).

For showing (2.2), we first compute the action of the first term right-hand side on some $v_{n} \otimes h_{n} \in \mathcal{D}_{n}(\tilde{\mathcal{H}})$. It is enough to do so on vectors of the form $v_{n}=e_{i} \otimes v_{n-1}$, $h_{n}=a \otimes h_{n-1}\left(a \in \mathcal{L}, v_{n-1} \otimes h_{n-1} \in \mathcal{D}_{n-1}(\tilde{\mathcal{H}})\right)$ as they form a total set in $\mathcal{H}^{\otimes n}$ and $\mathcal{L}^{\otimes n}$, respectively. We have: 


$$
\begin{aligned}
S_{\beta \delta}^{\alpha \gamma} z_{S, \gamma}^{*}(g) z_{S, \delta}(f) v_{n} \otimes h_{n} & =\sqrt{n} S_{\beta \delta}^{\alpha \gamma} z_{S, \gamma}^{*}(g)\left\langle e_{\delta} \otimes f\right|\left(e_{i} \otimes v_{n-1} \otimes a \otimes h_{n-1}\right) \\
& =n S_{\beta \delta}^{\alpha \gamma} \delta_{i}^{\delta}\langle f, a\rangle P_{n}^{S}\left(e_{\gamma} \otimes v_{n-1} \otimes g \otimes h_{n-1}\right) \\
& =n S_{\beta i}^{\alpha \gamma}\langle f, a\rangle P_{n}^{S}\left(e_{\gamma} \otimes v_{n-1} \otimes g \otimes h_{n-1}\right) .
\end{aligned}
$$

Since $v_{n-1}$ and $h_{n-1}$ are correctly symmetrised, the action of the projection $P_{n}^{S}$ in the final line simplifies. Namely, we need only sum over the permutations that shift the $e_{\gamma}$ and $g$ terms such that they appear in each tensor slot [13]; $P_{n}^{S}=\frac{1}{n} \sum_{k=1}^{n} D_{n}^{S}\left(\sigma_{k}\right)$. $1 \otimes P_{n-1}^{S}$. The above now reads

$$
S_{\beta \delta}^{\alpha \gamma} z_{S, \gamma}^{*}(g) z_{S, \delta}(f) v_{n} \otimes h_{n}=S_{\beta i}^{\alpha \gamma}\langle f, a\rangle \sum_{k=1}^{n} D_{n}^{S}\left(\sigma_{k}\right)\left(e_{\gamma} \otimes v_{n-1} \otimes g \otimes h_{n-1}\right)(3
$$

Recall that the left-hand side also depends on $e_{i}$ and $a$ via the definitions $v_{n}=e_{i} \otimes v_{n-1}$, $h_{n}=a \otimes h_{n-1}$. To compute the left-hand side of (2.2), we now consider its scalar product with a $w_{n} \otimes b_{n}:=e_{j} \otimes w_{n-1} \otimes c \otimes b_{n-1} \in \mathcal{D}_{n}(\tilde{\mathcal{H}})$ in the scalar product:

$$
\begin{aligned}
& \left\langle w_{n} \otimes b_{n}, z_{S, \alpha}(f) z_{S, \beta}^{*}(g) v_{n} \otimes h_{n}\right\rangle=\left\langle z_{S, \alpha}^{*}(f) w_{n} \otimes b_{n}, z_{S, \beta}^{*}(g) v_{n} \otimes h_{n}\right\rangle \\
& =(n+1)\left\langle e_{\alpha} \otimes w_{n} \otimes f \otimes b_{n}, P_{n+1}^{S}\left(e_{\beta} \otimes v_{n} \otimes g \otimes h_{n}\right)\right\rangle .
\end{aligned}
$$

Since $v_{n}$ and $h_{n}$ are correctly symmetrised, the projection $P_{n+1}^{S}$ again simplifies as before. Noting further that $\sigma_{1}=1$, this gives:

$$
\begin{aligned}
\left\langle w_{n} \otimes b_{n}, z_{S, \alpha}(f) z_{S, \beta}^{*}(g) v_{n} \otimes h_{n}\right\rangle & \\
= & \left\langle e_{\alpha} \otimes w_{n} \otimes f \otimes b_{n}, \sum_{k=1}^{n+1} D_{n+1}^{S}\left(\sigma_{k}\right)\left(e_{\beta} \otimes v_{n} \otimes g \otimes h_{n}\right)\right\rangle \\
= & \left\langle e_{\alpha} \otimes w_{n} \otimes f \otimes b_{n},\left[1+\sum_{k=2}^{n+1} D_{n+1}^{S}\left(\sigma_{k}\right)\right]\left(e_{\beta} \otimes v_{n} \otimes g \otimes h_{n}\right)\right\rangle \\
= & \delta_{\beta}^{\alpha}\langle f, g\rangle\left\langle w_{n} \otimes b_{n}, v_{n} \otimes h_{n}\right\rangle \\
& +\left\langle e_{\alpha} \otimes w_{n} \otimes f \otimes b_{n}, \sum_{k=2}^{n+1} D_{n+1}^{S}\left(\sigma_{k}\right)\left(e_{\beta} \otimes e_{i} \otimes v_{n-1} \otimes g \otimes a \otimes h_{n-1}\right)\right\rangle .
\end{aligned}
$$

To shift the index of the sum $\sum_{k=2}^{n+1} D_{n+1}^{S}\left(\sigma_{k}\right)$, we note that it sums over the permutations shifting the $e_{\beta}$ term through each tensor slot with the first term being the permutation given by just $S_{1, n+1} \otimes F_{1}=D_{n+1}^{S}\left(\tau_{1}\right)$. If we extract this term from the sum, we can read the remaining terms as taking the second tensor slot and permuting through the remaining $n$ slots with the first slot being untouched [13]. Concretely, this means that we can write this as

$$
\sum_{k=2}^{n+1} D_{n+1}^{S}\left(\sigma_{k}\right)=\left(\sum_{k=1}^{n} 1 \otimes D_{n}^{S}\left(\sigma_{k}\right)\right) D_{n+1}^{S}\left(\tau_{1}\right)
$$


which we use and continue in the calculation:

$$
\begin{aligned}
\left\langle w_{n} \otimes b_{n}, z_{S, \alpha}(f) z_{S, \beta}^{*}(g) v_{n} \otimes h_{n}\right\rangle=\delta_{\beta}^{\alpha}\langle f, g\rangle\left\langle w_{n} \otimes b_{n}, v_{n} \otimes h_{n}\right\rangle \\
\quad+S_{\beta i}^{\xi \gamma}\left\langle e_{\alpha} \otimes e_{j} \otimes w_{n-1} \otimes f \otimes b_{n}, \sum_{k=1}^{n} 1 \otimes D_{n}^{S}\left(\sigma_{k}\right)\left(e_{\xi} \otimes e_{\gamma} \otimes v_{n-1} \otimes a \otimes g \otimes h_{n-1}\right)\right\rangle \\
=\delta_{\beta}^{\alpha}\langle f, g\rangle\left\langle w_{n} \otimes b_{n}, v_{n} \otimes h_{n}\right\rangle \\
\quad+S_{\beta i}^{\alpha \gamma}\langle f, a\rangle\left\langle w_{n} \otimes b_{n}, \sum_{k=1}^{n} D_{n}^{S}\left(\sigma_{k}\right)\left(e_{\gamma} \otimes v_{n-1} \otimes g \otimes h_{n-1}\right)\right\rangle .
\end{aligned}
$$

Since all elements involved were arbitrary, we now read off what we have computed by comparing the right-hand slot of the scalar product:

$$
\begin{aligned}
z_{S, \alpha}(f) z_{S, \beta}^{*}(g) v_{n} \otimes h_{n}= & \delta_{\beta}^{\alpha}\langle f, g\rangle v_{n} \otimes h_{n} \\
& +S_{\beta i}^{\alpha \gamma}\langle f, a\rangle \sum_{k=1}^{n} D_{n}^{S}\left(\sigma_{k}\right)\left(e_{\gamma} \otimes v_{n-1} \otimes g \otimes h_{n-1}\right) .
\end{aligned}
$$

We can now read that (up to the contraction term) we have equality between (3.9) and (3.10), and thus, (2.2) is satisfied so $\pi_{S}$ is indeed a representation of $\mathcal{Z}(S, \mathcal{L})$ on $U \mathcal{F}_{S}^{0}(\tilde{\mathcal{H}})$.

Moreover, $\Omega_{S}$ is cyclic for this representation by Proposition 3.3, and then, the GNS property follows once we realise that the functional defined by the right-hand side of (3.8) satisfies the properties of $\omega$ as outlined in Definition 2.1 since $\Omega_{S}$ is a normalised vector and $z_{S}$ annihilates it.

Remark 3.5 It is now apparent that $\omega$ is positive (for $S \in \mathcal{R}_{0}(\mathcal{H})$ ). For any $X \in$ $\mathcal{Z}(S, \mathcal{L})$ :

$$
\begin{aligned}
\omega\left(X^{*} X\right) & =\left\langle\Omega_{S}, \pi_{S}\left(X^{*} X\right) \Omega_{S}\right\rangle \\
& =\left\langle\Omega_{S}, \pi_{S}(X)^{*} \pi_{S}(X) \Omega_{S}\right\rangle \\
& =\left\langle\pi_{S}(X) \Omega_{S}, \pi_{S}(X) \Omega_{S}\right\rangle \\
& =\left\|\pi_{S}(X) \Omega_{S}\right\|^{2} \geq 0 .
\end{aligned}
$$

Revisiting the examples outlined in the previous section, we see that for $S_{\delta \gamma}^{\alpha \beta}= \pm \delta_{\gamma}^{\alpha} \delta_{\delta}^{\beta}$ we arrive at the totally symmetric (+) or totally anti-symmetric (-) Fock space over $\tilde{\mathcal{H}}$, usually known as the Bosonic and Fermionic Fock spaces, respectively.

The case of $S_{\delta \gamma}^{\alpha \beta}=-\delta_{\delta}^{\alpha} \delta_{\gamma}^{\beta}(S=-1)$ results in a very small space for $\mathcal{L}=\mathbb{C}$ : For this particular choice of $S$, the projection simplifies greatly to

$$
P_{n}^{S}=\sum_{\pi \in \mathfrak{S}_{n}} \operatorname{sgn}(\pi)= \begin{cases}1, & n=1 \\ 0, & n>1\end{cases}
$$


where $\operatorname{sgn}(\pi)$ is the sign of the permutation $\pi$. There then only exists a zero-particle space (multiples of the vacuum) and the single particle space $\tilde{\mathcal{H}}$ :

$$
\mathcal{F}_{S}(\tilde{\mathcal{H}})=\Omega_{S} \oplus \tilde{\mathcal{H}}
$$

The other extreme is given by $S=1$ and $\mathcal{L}=\mathbb{C}$, which generates the full unsymmetrised (or "Boltzmann") Fock space

$$
\mathcal{F}_{S}(\mathcal{H})=\bigoplus_{n \geq 0} \mathcal{H}^{\otimes n}
$$

In general, $\mathcal{F}_{S}(\mathcal{H})$ “interpolates" between these two extreme cases.

\section{Operations on $\boldsymbol{R}$-matrices and equivalences}

In this work, we aim to generalise the following notion (sometimes referred to as an "exponential relation", see, for example, [4] and references therein): Let $\mathcal{H}, \mathcal{K}$ be Hilbert spaces, and denote by $\mathcal{F}_{ \pm}(\mathcal{H})$ the Bosonic/Fermionic Fock space over $\mathcal{H}$. To keep touch with our earlier constructions, this corresponds to the Fock representation of the algebra $\mathcal{Z}\left( \pm F_{\mathcal{H}}, \mathbb{C}\right.$ ), where $F_{\mathcal{H}}$ is the tensor flip on $\mathcal{H}^{\otimes 2}$ (we simplify notation here using \pm as a subscript to mean $S= \pm F_{\mathcal{H}}$ to remain familiar with notation existing in the literature). Then it is well known that there exists a natural isomorphism

$$
\mathcal{F}_{ \pm}(\mathcal{H} \oplus \mathcal{K}) \cong \mathcal{F}_{ \pm}(\mathcal{H}) \otimes \mathcal{F}_{ \pm}(\mathcal{K})
$$

This isomorphism does not only hold as a Hilbert space isomorphism (which is trivial for the case of infinite-dimensional separable Hilbert spaces), but also as an isomorphism of representations of the CCR/CAR algebras.

In the following, we will explore to which extent (4.1) generalises to our setting of $S$-symmetric Fock spaces. As a prerequisite for doing so, we need to compare $R$ matrices on tensor products and direct sums of Hilbert spaces. The relevant notions are recalled below.

Definition 4.1 [16] Let $\mathcal{H}, \mathcal{K}$ be Hilbert spaces, and $S \in \mathcal{R}_{0}(\mathcal{H}), R \in \mathcal{R}_{0}(\mathcal{K})$. Then we define

(i) $S \boxplus R:(\mathcal{H} \oplus \mathcal{K}) \otimes(\mathcal{H} \oplus \mathcal{K}) \rightarrow(\mathcal{H} \oplus \mathcal{K}) \otimes(\mathcal{H} \oplus \mathcal{K})$ as

$$
\begin{aligned}
& S \boxplus R:=S \oplus R \oplus F, \quad \text { on } \\
& (\mathcal{H} \oplus \mathcal{K}) \otimes(\mathcal{H} \oplus \mathcal{K})=(\mathcal{H} \otimes \mathcal{H}) \oplus(\mathcal{K} \otimes \mathcal{K}) \oplus(\mathcal{H} \otimes \mathcal{K}) \oplus(\mathcal{K} \otimes \mathcal{H}) .
\end{aligned}
$$

(ii) $S \otimes R: \mathcal{H} \otimes \mathcal{K} \otimes \mathcal{H} \otimes \mathcal{K} \rightarrow \mathcal{H} \otimes \mathcal{K} \otimes \mathcal{H} \otimes \mathcal{K}$ as

$$
S \otimes R=F_{2}(S \otimes R) F_{2}
$$

where $F_{2}$ exchanges the second and third tensor factors. 
We use the terminology box-sum and box-product, respectively, for these operations.

These two operations preserve unitarity, involutivity and if $S, R$ are solutions to the Yang-Baxter equation, then $S \boxplus R, S \otimes R$ are also solutions, and hence, we have that $S \boxplus R \in \mathcal{R}_{0}(\mathcal{H} \oplus \mathcal{K})$ and $S \otimes R \in \mathcal{R}_{0}(\mathcal{H} \otimes \mathcal{K})$ [16].

A generalisation of (4.1) that we will show in this work (for now setting $\mathcal{L}=\mathbb{C}$ ) now reads as

$$
\mathcal{F}_{S \boxplus R}(\mathcal{H} \oplus \mathcal{K}) \cong \mathcal{F}_{S}(\mathcal{H}) \otimes \mathcal{F}_{R}(\mathcal{K})
$$

where $S \in \mathcal{R}_{0}(\mathcal{H}), R \in \mathcal{R}_{0}(\mathcal{K})$ for Hilbert spaces $\mathcal{H}, \mathcal{K}$. Setting $S=F_{\mathcal{H}}, R=$ $F_{\mathcal{K}}$, then $S \boxplus R=F_{\mathcal{H} \oplus \mathcal{K}}$ and the above reads the same as (4.1) for the Bose case, so we at least realise immediately this is consistent with existing results.

Under the assumption that all resulting Fock spaces are finite-dimensional, isomorphism (4.2) has recently been established by Pennig using exponential functors [21]. This assumption of finite dimensionality is satisfied, for example, by $S=-1$ or $S=-F_{\mathcal{H}}$, and more generally for all $R$-matrices having only "Fermionic" Thoma parameters. For applications to quantum field theory, however, it is essential to have an infinite-dimensional Hilbert space, and our setup and arguments in the following will be quite different from that of [21].

In addition, the analysis of equivalent functors in [21] also yielded an equivalence between functors when there is a natural equivalence between the $R$-matrices associated with them. We may also wonder if it is possible to formulate such an isomorphism in our setting and to this end, we recall the notion of equivalent $R$-matrices.

Definition 4.2 [16] Let $\mathcal{H}, \mathcal{K}$ be Hilbert spaces, $S \in \mathcal{R}_{0}(\mathcal{H}), R \in \mathcal{R}_{0}(\mathcal{K})$, then they are said to be equivalent - denoted as $S \sim R$-if and only if, for each $n \in \mathbb{N}$ the representations $D_{n}^{S}$ and $D_{n}^{R}$ are unitarily equivalent.

Let $S \in \mathcal{R}_{0}(\mathcal{H}), R \in \mathcal{R}_{0}(\mathcal{K})$. If $S \sim R$, this definition means that there exists a unitary intertwining operator $Y_{n}^{S, R}: \mathcal{H}_{n} \rightarrow \mathcal{K}_{n}$ such that

$$
Y_{n}^{S, R} D_{n}^{S}(\pi)=D_{n}^{R}(\pi) Y_{n}^{S, R} . \quad\left(\pi \in \mathfrak{S}_{n}\right)
$$

In general, the form of $Y_{n}^{S, R}$ is unknown, but we can provide a few examples [16] of when we may write down its action explicitely.

- Type 1: There exists a unitary $Q$ on $\mathcal{H}$ such that $(Q \otimes Q) S\left(Q^{*} \otimes Q^{*}\right)=R$. Then $S \sim R$ and we may choose

$$
Y_{n}^{S, R}=Q^{\otimes n}
$$


- Type 2: There exists a unitary $Q$ on $\mathcal{H}$ such that $[S, Q \otimes Q]=0$ and $(1 \otimes Q) S(1 \otimes$ $\left.Q^{*}\right)=R$. Then $S \sim R$ and we may choose

$$
Y_{n}^{S, R}=1 \otimes Q \otimes \cdots \otimes Q^{n-1}
$$

- Type 3: Let $F: \mathcal{H}^{\otimes 2} \rightarrow \mathcal{H}^{\otimes 2}, F(x \otimes y)=y \otimes x,(x, y \in \mathcal{H})$ be the "flip" operator, such that $F S F=R$. Then $S \sim R$ and we may choose

$$
Y_{n}^{S, R}=D_{n}^{F S F}\left(\iota_{n}\right)^{-1} D_{n}^{F}\left(\iota_{n}\right)
$$

where $\iota_{n}$ is the total inversion permutation in $n$ letters.

The significance of the equivalence relation $\sim$ stems from the fact that every $S \in \mathcal{R}_{0}(\mathcal{H})$ is equivalent to a very simple $R$-matrix, namely an $R$-matrix of socalled normal form: It was shown in [16] that for any $S \in \mathcal{R}_{0}(\mathcal{H})$, there exists $N \in \mathbb{N}$, dimension parameters $d_{1}, \ldots, d_{N} \in \mathbb{N}$ with $d_{1}+\cdots+d_{N}=d_{\mathcal{H}}$ and signs $\varepsilon_{1}, \ldots, \varepsilon_{N} \in\{+1,-1\}$, such that

$$
S \sim \bigoplus_{i=1}^{N} \varepsilon_{i} 1_{d_{i}^{2}}
$$

From this definition, we can read off that the tensor flip $F_{\mathcal{H}}=\boxplus_{i=1}^{d_{\mathcal{H}}} 1$ is a normal form, and also the identity (take $N=1$ and $\varepsilon_{1}=+1$ ), as examples. Considering now two equivalent $R$-matrices $S \sim R$, we may wonder whether we have an isomorphism

$$
\mathcal{F}_{S}(\mathcal{H}) \cong \mathcal{F}_{R}(\mathcal{K})
$$

where this could simply be an isomorphism of Hilbert spaces, or even an isomorphism of representations of $\mathrm{ZF}$ algebras.

Since any $R$-matrix $S$ is equivalent to a normal form (4.4), the combination of the anticipated isomorphisms (4.5) and (4.2) would allow us to split $\mathcal{F}_{S}(\mathcal{H})$ into a tensor product of Fock spaces of the simple form $\mathcal{F}_{ \pm 1}\left(\mathcal{H}_{i}\right)$.

As a preparatory step to the next section where we cement these ideas, we note the following results.

Lemma 4.3 Let $\mathcal{H}, \mathcal{K}$ be separable Hilbert spaces, and $S \in \mathcal{R}_{0}(\mathcal{H}), R \in \mathcal{R}_{0}(\mathcal{K})$. Then the representation of the symmetric group, $D_{n}^{S \bigotimes R}$, generated by $S \otimes R$ is unitarily equivalent to $D_{n}^{S} \otimes D_{n}^{R}$ for any $n \in \mathbb{N}$.

Proof We show the result for only the generating elements $\tau_{k}$, of $\mathfrak{S}_{n}$. Let $\left(h_{\alpha}\right)_{\alpha \in \mathbb{N}}$ and $\left(k_{\beta}\right)_{\beta \in \mathbb{N}}$ be orthonormal bases of $\mathcal{H}$ and $\mathcal{K}$ respectively. Employing the operator $U_{n}$, an element in the domain of $D_{n}^{S \bigotimes R}$ is mapped to an element in the domain of $D_{n}^{S} \otimes D_{n}^{R}$ and the action of the latter operator is given by 


$$
\begin{aligned}
& D_{n}^{S}\left(\tau_{k}\right) \otimes D_{n}^{R}\left(\tau_{k}\right)\left(\bigotimes_{i=1}^{n} h_{\alpha_{i}}\right) \otimes\left(\bigotimes_{i=1}^{n} k_{\beta_{i}}\right) \\
& =S_{\delta \gamma}^{\alpha_{k} \alpha_{k+1}} R_{\eta \xi}^{\beta_{k} \beta_{k+1}}\left(\left(\bigotimes_{i=1}^{k-1} h_{\alpha_{i}}\right) \otimes h_{\delta} \otimes h_{\gamma} \otimes\left(\bigotimes_{i=k+2}^{n} h_{\alpha_{i}}\right)\right) \\
& \quad \otimes\left(\left(\bigotimes_{i=1}^{k-1} k_{\beta_{i}}\right) \otimes k_{\eta} \otimes k_{\xi} \otimes\left(\bigotimes_{i=k+2}^{n} k_{\beta_{i}}\right)\right),
\end{aligned}
$$

where the implicit sums converge in norm topology. Applying the linear operator $U_{n}^{*}$ to the above gives the action of $D_{n}^{S \bigotimes R}$ as stated.

Corollary 4.4 Let $\mathcal{H}_{1}, \mathcal{H}_{2}, \mathcal{K}_{1}, \mathcal{K}_{2}$ be Hilbert spaces, $S_{1} \in \mathcal{R}_{0}\left(\mathcal{H}_{1}\right), R_{1} \in \mathcal{R}_{0}\left(\mathcal{K}_{1}\right)$, $S_{2} \in \mathcal{R}_{0}\left(\mathcal{H}_{2}\right), R_{2} \in \mathcal{R}_{0}\left(\mathcal{K}_{2}\right)$ such that $S_{1} \sim S_{2}$ and $R_{1} \sim R_{2}$. Then an intertwiner for $S_{1} \otimes R_{1} \sim S_{2} \otimes R_{2}, Y^{S_{1} \bigotimes R_{1}, S_{2} \otimes R_{2}}$, is given by

$$
Y_{n}^{S_{1} \otimes R_{1}, S_{2} \otimes R_{2}}=U_{n}^{*}\left(Y_{n}^{S_{1}, S_{2}} \otimes Y_{n}^{R_{1}, R_{2}}\right) U_{n}
$$

with $Y^{S_{1}, S_{2}}, Y^{R_{1}, R_{2}}$ intertwiners between $S_{1}, S_{2}$ and $R_{1}, R_{2}$, respectively.

Proof This is clear from the definition of $U_{n}$ and Lemma 4.3.

We mention as an aside that an analogue of Corollary 4.4 also holds for box-sums $S_{1} \boxplus R_{1} \sim S_{2} \boxplus R_{2}$. As we will not need this here, we omit the details.

\section{Isomorphisms between polynomial algebras and equivalences of representations}

We now go on to discuss generalisations of (4.1), and in particular, we consider (4.2) with the addition of the Hilbert space $\mathcal{L}$ appearing in a tensor product with both $\mathcal{H}, \mathcal{K}$. For $S \in \mathcal{R}_{0}(\mathcal{H}), R \in \mathcal{R}_{0}(\mathcal{K})$, define $S \otimes F=: \tilde{S} \in \mathcal{R}_{0}(\tilde{\mathcal{H}})$, for $F$ the tensor flip on $\mathcal{L} \otimes \mathcal{L}$, and similarly for $R$. As mentioned previously, the tilde appearing above $R$-matrices always signifies a box-product with $F$, and above a Hilbert space always means a tensor product with the same space $\mathcal{L}$. With this notation, we will now aim to show the following:

$$
\mathcal{F}_{\tilde{S} \boxplus \tilde{R}}(\tilde{\mathcal{H}} \oplus \tilde{\mathcal{K}}) \cong \mathcal{F}_{\tilde{S}}(\tilde{\mathcal{H}}) \otimes \mathcal{F}_{\tilde{R}}(\tilde{\mathcal{K}})
$$

On the left-hand side, the GNS representation is already described in Sect. 3, where we have a space symmetrised by the operator $\tilde{S} \boxplus \tilde{R}$, but so far we have not considered representations of Zamolodchikov operators on $\mathcal{F}_{\tilde{S}}(\tilde{\mathcal{H}}) \otimes \mathcal{F}_{\tilde{R}}(\tilde{\mathcal{K}})$. We will first build data on this space-most notably the analogue of the creation/annihilation operators and vacuum vector. The exchange relations between the former and the cyclicity of the latter will be shown, before a GNS-type argument will prove that they are in fact equivalent representations of the same algebra $\mathcal{Z}(S \boxplus R, \mathcal{L})$. 
We begin with the algebra $\mathcal{Z}(S \boxplus R, \mathcal{L})$ as given in Sect. 1, with $S \in \mathcal{R}_{0}(\mathcal{H}), R \in$ $\mathcal{R}_{0}(\mathcal{K})$ and note a property regarding distributivity of the box-product over the boxsum. Generally, the distributivity property is only known up to equivalence, but in the specific cases considered in this work, we show that we in fact have equality.

Lemma 5.1 Let $\mathcal{H}, \mathcal{K}$ be finite-dimensional Hilbert spaces, $S \in \mathcal{R}_{0}(\mathcal{H}), R \in \mathcal{R}_{0}(\mathcal{K})$. Then

$$
(S \boxplus R) \otimes F=\tilde{S} \boxplus \tilde{R} .
$$

Proof On the level of the spaces they act on, we first note that

$$
\begin{aligned}
\mathcal{D}((S \boxplus R) \otimes F) & =((\mathcal{H} \oplus \mathcal{K}) \otimes \mathcal{L})^{\otimes 2} \\
& \cong(\tilde{\mathcal{H}} \oplus \tilde{\mathcal{K}})^{\otimes 2}=\mathcal{D}(\tilde{S} \boxplus \tilde{R}),
\end{aligned}
$$

where by $\mathcal{D}(S)$ we mean the domain of $S$.

To show they do indeed map vectors in their respective domains to the same vector, we consider each orthogonal component of their domains and discuss how each operator acts.

Firstly, the case of $(\mathcal{H} \otimes \mathcal{L})^{\otimes 2}$, the left-hand side of (5.2) first applies $F_{2}$ and then acts as a flip in the $\mathcal{L}^{\otimes 2}$ parts, and as $S \boxplus R$ on $\mathcal{H}^{\otimes 2}$ which by definition simply acts as just $S$, and finally applies a second $F_{2}$. More simply put, it flips the contributions from $\mathcal{L}$ and acts as $S$ on the contributions from $\mathcal{H}$. The same occurs on the right-hand side of (5.2), where we see that it simply acts as only $\tilde{S}$ by definition of the box-sum, which flips the contributions from $\mathcal{L}$ and acts as $S$ on the contributions from $\mathcal{H}$.

Similarly, for the case of $(\mathcal{K} \otimes \mathcal{L})^{\otimes 2}$, the left-hand side of (5.2) flips on $\mathcal{L}$ and acts as $R$ on $\mathcal{K}$. Identically, the right-hand side of (5.2) acts just as $\tilde{R}$ which again flips on $\mathcal{L}$ and acts as $\mathcal{K}$ and so we equality again.

The remaining cases to consider are $\mathcal{H} \otimes \mathcal{L} \otimes \mathcal{K} \otimes \mathcal{L}$ and $\mathcal{K} \otimes \mathcal{L} \otimes \mathcal{H} \otimes \mathcal{L}$. However, since we have a single contribution from both $\mathcal{H}$ and $\mathcal{K}$ appearing, each operator simply reduces to a combination of flips acting on the appropriate spaces and it is easy to realise that $F_{2}\left(F_{\mathcal{H} \oplus \mathcal{K}} \otimes F\right) F_{2}=F_{\tilde{\mathcal{H}} \otimes \tilde{\mathcal{K}}}$ and $F_{2}\left(F_{\mathcal{K} \oplus \mathcal{H}} \otimes F\right) F_{2}=F_{\tilde{\mathcal{K}} \otimes \tilde{\mathcal{H}}}$.

Both sides of (5.2) then act in the same way on each orthogonal part of their isomorphic domains, therefore they are equal.

The algebra of interest in this section, $\mathcal{Z}(S \boxplus R, \mathcal{L})$, is described by the operator $(S \boxplus R) \otimes F$, but now Lemma 5.1 allows us to work instead with $\tilde{S} \boxplus \tilde{R}$.

We consider the Fock space $\mathcal{F}_{\tilde{S} \boxplus \tilde{R}}(\tilde{\mathcal{H}} \oplus \tilde{\mathcal{K}})$ on which we have a vacuum vector $\Omega_{\tilde{S} \boxplus \tilde{R}}$ and creation/annihilation operators $z_{\tilde{S} \boxplus \tilde{R}}^{*}, z_{\tilde{S} \boxplus \tilde{R}}$. The latter obey exchange relations involving the operator $\tilde{S} \boxplus \tilde{R}$, which we note here for convenience. We adopt the shorthand notation $\tilde{\mathcal{H}} \ni f_{\alpha}:=e_{\alpha} \otimes f$ for $f \in \mathcal{L}$ and basis vectors $e_{\alpha}$ of $\mathcal{H}$, and $\tilde{\mathcal{K}} \ni g_{\xi}:=k_{\xi} \otimes g$ for $g \in \mathcal{L}$ and basis vectors $k_{\xi}$ of $\mathcal{K}$. Then

$$
\begin{gathered}
z_{\tilde{S} \boxplus \tilde{R}}\left(f_{\alpha} \oplus 0\right) z_{\tilde{S} \boxplus \tilde{R}}\left(g_{\beta} \oplus 0\right)=S_{\delta \gamma}^{\beta \alpha} z_{\tilde{S} \boxplus \tilde{R}}\left(g_{\gamma} \oplus 0\right) z_{\tilde{S} \boxplus \tilde{R}}\left(f_{\delta} \oplus 0\right), \\
z_{\tilde{S} \boxplus \tilde{R}}\left(f_{\alpha} \oplus 0\right) z_{\tilde{S} \boxplus \tilde{R}}^{*}\left(g_{\beta} \oplus 0\right)=S_{\beta \delta}^{\alpha \gamma} z_{\tilde{S} \boxplus \tilde{R}}^{*}\left(g_{\gamma} \oplus 0\right) z_{\tilde{S} \boxplus \tilde{R}}\left(f_{\delta} \oplus 0\right)+\delta_{\beta}^{\alpha}\langle f, g\rangle \cdot 1_{\tilde{\mathcal{H}} \oplus \tilde{\mathcal{K}}},
\end{gathered}
$$




$$
\begin{gathered}
z_{\tilde{S} \boxplus \tilde{R}}\left(0 \oplus f_{\xi}\right) z_{\tilde{S} \boxplus \tilde{R}}\left(0 \oplus g_{\eta}\right)=R_{\epsilon \pi}^{\eta \xi} z_{\tilde{S} \boxplus \tilde{R}}\left(0 \oplus g_{\pi}\right) z_{\tilde{S} \boxplus \tilde{R}}\left(0 \oplus f_{\epsilon}\right), \\
z_{\tilde{S} \boxplus \tilde{R}}\left(0 \oplus f_{\xi}\right) z_{\tilde{S} \boxplus \tilde{R}}^{*}\left(0 \oplus g_{\eta}\right)=R_{\eta \epsilon}^{\xi \pi} z_{\tilde{S} \boxplus \tilde{R}}^{*}\left(0 \oplus g_{\pi}\right) z_{\tilde{S} \boxplus \tilde{R}}\left(0 \oplus f_{\epsilon}\right)+\delta_{\eta}^{\xi}\langle f, g\rangle \cdot 1_{\tilde{\mathcal{H}} \oplus \tilde{\mathcal{K}}}, \\
z_{\tilde{S} \boxplus \tilde{R}}\left(f_{\alpha} \oplus 0\right) z_{\tilde{S} \boxplus \tilde{R}}\left(0 \oplus g_{\eta}\right)=z_{\tilde{S} \boxplus \tilde{R}}\left(0 \oplus g_{\eta}\right) z_{\tilde{S} \boxplus \tilde{R}}\left(f_{\alpha} \oplus 0\right) \\
z_{\tilde{S} \boxplus \tilde{R}}\left(f_{\alpha} \oplus 0\right) z_{\tilde{S} \boxplus \tilde{R}}^{*}\left(0 \oplus g_{\eta}\right)=z_{\tilde{S} \boxplus \tilde{R}}^{*}\left(0 \oplus g_{\eta}\right) z_{\tilde{S} \boxplus \tilde{R}}\left(f_{\alpha} \oplus 0\right)
\end{gathered}
$$

These operators along with the identity $1_{\tilde{\mathcal{H}} \oplus \tilde{\mathcal{K}}}$ generate the polynomial algebra $\mathcal{P}_{\tilde{S} \boxplus \tilde{R}}$ and form our natural Fock representation of the algebra $\mathcal{Z}(S \boxplus R, \mathcal{L})$.

We now consider a tensor product of Fock spaces

$$
\mathcal{F}_{\tilde{S}}(\tilde{\mathcal{H}}) \otimes \mathcal{F}_{\tilde{R}}(\tilde{\mathcal{K}})
$$

and on each Fock space, we have creation/annihilation operators $z_{\tilde{S}}^{*}, z_{\tilde{S}}, z_{\tilde{R}}^{*}, z_{\tilde{R}}$ acting endomorphically on $\mathcal{F}_{\tilde{S}}^{0}(\tilde{\mathcal{H}}), \mathcal{F}_{\tilde{R}}^{0}(\tilde{\mathcal{K}})$ respectively; vacuum vectors $\Omega_{\tilde{S}}, \Omega_{\tilde{R}}$ define similar data on $\mathcal{F}_{\tilde{S}}(\tilde{\mathcal{H}}) \otimes \mathcal{F}_{\tilde{R}}(\tilde{\mathcal{K}})$ :

$$
\begin{gathered}
z_{\tilde{S}, \tilde{R}}\left(f_{\alpha} \oplus g_{\xi}\right):=z_{\tilde{S}, \alpha}(f) \otimes 1_{\tilde{\mathcal{K}}}+1_{\tilde{\mathcal{H}}} \otimes z_{\tilde{R}, \xi}(g), \quad f, g \in \mathcal{L}, \\
\Omega_{\tilde{S}, \tilde{R}}:=\Omega_{\tilde{S}} \otimes \Omega_{\tilde{R}} .
\end{gathered}
$$

The polynomial algebra $\mathcal{P}_{\tilde{S}, \tilde{R}}$ is then defined as the algebra generated by the operators $1_{\tilde{\mathcal{H}}} \otimes 1_{\tilde{\mathcal{K}}}, z_{\tilde{S}, \tilde{R}}^{*}, z_{\tilde{S}, \tilde{R}}$

Lemma 5.2 The vacuum vector $\Omega_{\tilde{S}, \tilde{R}}$ is cyclic for the polynomial algebra $\mathcal{P}_{\tilde{S}, \tilde{R}}$. That is, $\mathcal{P}_{\tilde{S}, \tilde{R}} \Omega_{\tilde{S}, \tilde{R}}$ is dense in $\mathcal{F}_{\tilde{S}}(\tilde{\mathcal{H}}) \otimes \mathcal{F}_{\tilde{R}}(\tilde{\mathcal{K}})$.

Proof Let $\psi \in \mathcal{F}_{\tilde{S}}(\tilde{\mathcal{H}}) \otimes \mathcal{F}_{\tilde{R}}(\tilde{\mathcal{K}})$ be orthogonal to $\mathcal{P}_{\tilde{S}, \tilde{R}} \Omega_{\tilde{S}, \tilde{R}}$. Then for any $i, j \in \mathbb{N}_{0}$ and vectors $f_{1}, \ldots, f_{i} \in \tilde{\mathcal{H}}, g_{1}, \ldots, g_{j} \in \tilde{\mathcal{K}}$

$$
\begin{aligned}
& \left\langle\psi_{i, j}, z_{\tilde{S}, \tilde{R}}^{*}\left(f_{1} \oplus 0\right) \cdots z_{\tilde{S}, \tilde{R}}^{*}\left(f_{i} \oplus 0\right) z_{\tilde{S}, \tilde{R}}^{*}\left(0 \oplus g_{1}\right) \cdots z_{\tilde{S}, \tilde{R}}^{*}\left(0 \oplus g_{j}\right) \Omega_{\tilde{S}} \otimes \Omega_{\tilde{R}}\right\rangle \\
& \quad=\sqrt{i ! j !}\left\langle\psi_{i, j}, P_{i}^{\tilde{S}} \otimes P_{j}^{\tilde{R}}\left(f_{1} \otimes \cdots \otimes f_{i} \otimes g_{1} \otimes \cdots \otimes g_{j}\right)\right\rangle \\
& \quad=\sqrt{i ! j !}\left\langle\psi_{i, j}, f_{1} \otimes \cdots \otimes f_{i} \otimes g_{1} \otimes \cdots \otimes g_{j}\right\rangle
\end{aligned}
$$

where $\psi_{i, j}$ is the $i, j$ th component of $\psi$, each letter corresponding to each tensor slot in $\mathcal{F}_{\tilde{S}}(\tilde{\mathcal{H}}) \otimes \mathcal{F}_{\tilde{R}}(\tilde{\mathcal{K}})$ and we have used that the self-adjoint projection $P_{i}^{\tilde{S}} \otimes P_{j}^{\tilde{R}}$ leaves the vector $\psi$ invariant. By the definition of the tensor product, the vectors $f_{1} \otimes \cdots \otimes f_{i} \otimes g_{1} \otimes \cdots \otimes g_{j}$ form a total set in $\tilde{\mathcal{H}}^{\otimes i} \otimes \tilde{\mathcal{K}}^{\otimes j}$, and hence, we conclude that $\psi=0$.

We are now ready to prove the claimed isomorphism of Fock spaces as representations of $\mathcal{Z}(S \boxplus R, \mathcal{L})$.

Theorem 5.3 Let $\mathcal{H}, \mathcal{K}$ be Hilbert spaces of finite dimensions $d_{\mathcal{H}}, d_{\mathcal{K}}$, respectively, and $\tilde{S} \in \mathcal{R}_{0}(\tilde{\mathcal{H}}), \tilde{R} \in \mathcal{R}_{0}(\tilde{\mathcal{K}})$, then: 
a) The map $\pi_{\tilde{S}, \tilde{R}}: \mathcal{Z}(S \boxplus R, \mathcal{L}) \rightarrow \mathcal{P}_{\tilde{S}, \tilde{R}}$

$$
\begin{aligned}
\pi_{\tilde{S}, \tilde{R}}\left(1_{\mathcal{Z}(S \boxplus R, \mathcal{L})}\right) & :=1_{\tilde{\mathcal{H}} \otimes \tilde{\mathcal{K}}}, \\
\pi_{\tilde{S}, \tilde{R}}\left(Z_{\alpha}(f)\right) & := \begin{cases}z_{\tilde{S}, \tilde{R}}\left(f_{\alpha} \oplus 0\right), & \alpha \in\left\{1, \ldots, d_{\mathcal{H}}\right\} \\
z_{\tilde{S}, \tilde{R}}\left(0 \oplus f_{\alpha-d_{\mathcal{H}}}\right), & \alpha \in\left\{d_{\mathcal{H}}+1, \ldots, d_{\mathcal{H}}+d_{\mathcal{K}}\right\}\end{cases}
\end{aligned}
$$

extends to a unital $*$-representation of $\mathcal{Z}(S \boxplus R, \mathcal{L})$ on $\mathcal{F}_{\tilde{S}}^{0}(\tilde{\mathcal{H}}) \otimes \mathcal{F}_{\tilde{R}}^{0}(\tilde{\mathcal{K}})$ with cyclic vector $\Omega_{\tilde{S}, \tilde{R}}$ and

$$
\omega_{\tilde{S}, \tilde{R}}(X)=\left\langle\Omega_{\tilde{S}, \tilde{R}}, \pi_{\tilde{S}, \tilde{R}}(X) \Omega_{\tilde{S}, \tilde{R}}\right\rangle, \quad X \in \mathcal{Z}(S \boxplus R, \mathcal{L}) .
$$

b) There exists a unitary $V: \mathcal{F}_{\tilde{S} \boxplus \tilde{R}}(\tilde{\mathcal{H}} \oplus \tilde{\mathcal{K}}) \rightarrow \mathcal{F}_{\tilde{S}}(\tilde{\mathcal{H}}) \otimes \mathcal{F}_{\tilde{R}}(\tilde{\mathcal{K}})$ such that

$$
V \Omega_{\tilde{S} \boxplus \tilde{R}}=\Omega_{\tilde{S}, \tilde{R}}, \quad V \pi_{\tilde{S} \boxplus \tilde{R}}(X) V^{*}=\pi_{\tilde{S}, \tilde{R}}(X), \quad(X \in \mathcal{Z}(S \boxplus R, \mathcal{L})) .
$$

Proof a) We show first that the operators $z_{\tilde{S}, \tilde{R}}^{*}, z_{\tilde{S}, \tilde{R}}$ satisfy the same relations as $z_{\tilde{S} \boxplus \tilde{R}}^{*}, z_{\tilde{S} \boxplus \tilde{R}}$ as outlined in (5.3)-(5.8), firstly noting that

$$
z_{\tilde{S}, \tilde{R}}\left(f_{\alpha} \oplus 0\right)=z_{\tilde{S}, \alpha}(f) \otimes 1_{\tilde{\mathcal{K}}}, \quad z_{\tilde{S}, \tilde{R}}\left(0 \oplus f_{\xi}\right)=1_{\tilde{\mathcal{K}}} \otimes z_{\tilde{R}, \xi}(f), \quad(f \in \mathcal{L}),
$$

and similarly for $z_{\tilde{S}, \tilde{R}}^{*}$.

Furthermore, the operators $z_{\tilde{S}}^{*}, z_{\tilde{S}}$ and $z_{\tilde{R}}^{*}, z_{\tilde{R}}$ satisfy exchange relations (2.1), (2.2) governed by $S$ and $R$, respectively. Let $f, g \in \mathcal{L}$ then

$$
\begin{aligned}
z_{\tilde{S}, \tilde{R}}\left(f_{\alpha} \oplus 0\right) z_{\tilde{S}, \tilde{R}}\left(g_{\beta} \oplus 0\right) & =z_{\tilde{S}, \alpha}(f) z_{\tilde{S}, \beta}(g) \otimes 1_{\tilde{\mathcal{K}}} \\
& =S_{\delta \gamma}^{\beta \alpha} z_{\tilde{S}, \gamma}(g) z_{\tilde{S}, \delta}(f) \otimes 1_{\tilde{\mathcal{K}}} \\
& =S_{\delta \gamma}^{\beta \alpha} z_{\tilde{S}, \tilde{R}}\left(g_{\gamma} \oplus 0\right) z_{\tilde{S}, \tilde{R}}\left(f_{\delta} \oplus 0\right),
\end{aligned}
$$

which gives (5.3). Relation (5.5) follows in an analogous way on the second tensor factor applying (2.1) for $z_{\tilde{R}}$. Similarly

$$
\begin{aligned}
z_{\tilde{S}, \tilde{R}}\left(f_{\alpha} \oplus 0\right) z_{\tilde{S}, \tilde{R}}^{*}\left(g_{\beta} \oplus 0\right) & =z_{\tilde{S}, \alpha}(f) z_{\tilde{S}, \beta}^{*}(g) \otimes 1_{\tilde{\mathcal{K}}} \\
& =S_{\beta \delta}^{\alpha \gamma} z_{\tilde{S}, \gamma}^{*}(g) z_{\tilde{S}, \delta}(f) \otimes 1_{\tilde{\mathcal{K}}}+\delta_{\beta}^{\alpha}\langle f, g\rangle 1_{\tilde{\mathcal{H}}} \otimes 1_{\tilde{\mathcal{K}}} \\
& =S_{\beta \delta}^{\alpha \gamma} z_{\tilde{S}, \tilde{R}}^{*}\left(g_{\gamma} \oplus 0\right) z_{\tilde{S}, \tilde{R}}\left(f_{\delta} \oplus 0\right)+\delta_{\beta}^{\alpha}\langle f, g\rangle 1_{\tilde{\mathcal{H}}} \otimes 1_{\tilde{\mathcal{K}}}
\end{aligned}
$$

As for (5.7), (5.8), we see that since $z_{\tilde{S}, \tilde{R}}\left(f_{\alpha} \oplus 0\right)$ and $z_{\tilde{S}, \tilde{R}}\left(0 \oplus g_{\eta}\right)$ operate on different tensor slots they commute. Cyclicity of the vacuum vector is shown in Lemma 5.2, and we note that the annihilation of the normalised vector $\Omega_{\tilde{S}, \tilde{R}}$ by $z_{\tilde{S}, \tilde{R}}$ shows that the functional defined by the right-hand side of (5.11) satisfies the properties listed in Definition 2.1 and therefore coincides with $\omega$. 
b) Let $X \in \mathcal{Z}(S \boxplus R, \mathcal{L})$. Then

$$
\begin{aligned}
\left\|\pi_{\tilde{S}, \tilde{R}}(X) \Omega_{\tilde{S}, \tilde{R}}\right\|^{2} & =\left\langle\pi_{\tilde{S}, \tilde{R}}(X) \Omega_{\tilde{S}, \tilde{R}}, \pi_{\tilde{S}, \tilde{R}}(X) \Omega_{\tilde{S}, \tilde{R}}\right\rangle \\
& =\left\langle\Omega_{\tilde{S}, \tilde{R}}, \pi_{\tilde{S}, \tilde{R}}\left(X^{*} X\right) \Omega_{\tilde{S}, \tilde{R}}\right\rangle \\
& =\omega\left(X^{*} X\right) \\
& =\left\|\pi_{\tilde{S} \boxplus \tilde{R}}(X) \Omega_{\tilde{S} \boxplus \tilde{R}}\right\|^{2} .
\end{aligned}
$$

This shows that the map $V: \mathcal{P}_{\tilde{S} \boxplus \tilde{S}} \Omega_{\tilde{S} \boxplus \tilde{R}} \rightarrow \mathcal{P}_{\tilde{S}, \tilde{R}} \Omega_{\tilde{S}, \tilde{R}}, V \pi_{\tilde{S} \boxplus \tilde{R}}(X) \Omega_{\tilde{S} \boxplus \tilde{R}}:=$ $\pi_{\tilde{S}, \tilde{R}}(X) \Omega_{\tilde{S}, \tilde{R}}(X \in \mathcal{Z}(S \boxplus R, \mathcal{L})$ is well defined and isometric. Moreover, for $X, Y \in \mathcal{Z}(S \boxplus R, \mathcal{L})$

$$
\begin{aligned}
V \pi_{\tilde{S} \boxplus \tilde{R}}(X) V^{*} \pi_{\tilde{S}, \tilde{R}}(Y) \Omega_{\tilde{S}, \tilde{R}} & =V \pi_{\tilde{S} \boxplus \tilde{R}}(X) \pi_{\tilde{S} \boxplus \tilde{R}}(Y) \Omega_{\tilde{S} \boxplus \tilde{R}} \\
& =V \pi_{\tilde{S} \boxplus \tilde{R}}(X Y) \Omega_{\tilde{S} \boxplus \tilde{R}} \\
& =\pi_{\tilde{S}, \tilde{R}}(X Y) \Omega_{\tilde{S}, \tilde{R}} \\
& =\pi_{\tilde{S}, \tilde{R}}(X) \pi_{\tilde{S}, \tilde{R}}(Y) \Omega_{\tilde{S}, \tilde{R}} .
\end{aligned}
$$

Since $\Omega_{\tilde{S} \boxplus \tilde{R}}$ is cyclic for the representation $\pi_{\tilde{S} \boxplus \tilde{R}}$ and $\Omega_{\tilde{S}, \tilde{R}}$ is cyclic for $\pi_{\tilde{S}, \tilde{R}}$, it follows that $V$ extends to a unitary $\mathcal{F}_{\tilde{S} \boxplus \tilde{R}}(\tilde{\mathcal{H}} \oplus \tilde{\mathcal{K}}) \rightarrow \mathcal{F}_{\tilde{S}}(\tilde{\mathcal{H}}) \otimes \mathcal{F}_{\tilde{R}}(\tilde{\mathcal{K}})$ satisfying (5.12).

As a corollary, we note the simple form of $S$-symmetrised Fock spaces in the case of $S$ a normal form as previously anticipated.

Corollary 5.4 Let $S \in R_{0}(\mathcal{H})$ be an involutive $R$-matrix of normal form (4.4). Then there is a unitary

$$
V: \mathcal{F}_{\tilde{S}}(\tilde{\mathcal{H}}) \rightarrow \bigotimes_{i=1}^{N} \mathcal{F}_{\tilde{\varepsilon}_{i}}\left(\tilde{\mathcal{H}}_{i}\right)
$$

where $\mathcal{H}=\bigoplus_{i=1}^{N} \mathcal{H}_{i}$.

By the results of [16], we know that any involutive $R$-matrix is equivalent to an $R$ matrix of normal form. This motivates to ask what we can say about the relation of the Hilbert spaces and the representations of $\mathcal{Z}(S, \mathcal{L}), \mathcal{Z}(R, \mathcal{L})$ for two equivalent $R$-matrices $S \sim R$. If they are equivalent, we have unitary intertwiners $Y_{n}^{\tilde{S}, \tilde{R}}$ (4.3), and their direct sum $Y^{\tilde{S}, \tilde{R}}=\bigoplus_{n} Y_{n}^{\tilde{S}, \tilde{R}}$ defines a unitary $\mathcal{F}_{\tilde{S}}(\tilde{\mathcal{H}}) \rightarrow \mathcal{F}_{\tilde{R}}(\tilde{\mathcal{K}})$.

In some cases, this Hilbert space isomorphism also intertwines the actions of the Zamolodchikov operators as we now discuss in two examples.

Example 5.5 Let $\tilde{S} \in \mathcal{R}_{0}(\tilde{\mathcal{H}}), \tilde{R} \in \mathcal{R}_{0}(\tilde{\mathcal{K}})$ with $S \sim R$ (in the type 1 sense) such that we may choose their intertwiner to be of the form

$$
Y_{n}^{S, R}=\left(Y_{1}^{S, R}\right)^{\otimes n}
$$


Then

$$
Y^{\tilde{S}, \tilde{R}} \pi_{\tilde{S}}\left(Z_{\alpha}(f)\right)\left(Y^{\tilde{S}, \tilde{R}}\right)^{*}=\pi_{\tilde{R}}\left(Y_{1}^{\tilde{S}, \tilde{R}} Z_{\alpha}(f)\right)
$$

Indeed, we can check by calculation-let $f \in \mathcal{L}$, then by Corollary 4.4 we have the expression $Y^{\tilde{S}, \tilde{R}}=U^{*}\left(Y^{S, R} \otimes 1\right) U$ where we note that the intertwiner acts trivially on $\mathcal{L}$. This gives

$$
\begin{aligned}
U^{*} & \left(Y^{S, R} \otimes 1\right) U z_{\tilde{S}, \alpha}(f) U^{*}\left(\left(Y^{S, R}\right)^{*} \otimes 1\right) U \\
& =U^{*}\left(Y^{S, R} \otimes 1\right) z_{S}\left(e_{\alpha}\right) \otimes z_{F}(f)\left(\left(Y^{S, R}\right)^{*} \otimes 1\right) U \\
& =U^{*}\left(Y^{S, R_{2}} z_{S}\left(e_{\alpha}\right)\left(Y^{S, R}\right)^{*} \otimes z_{F}(f)\right) U
\end{aligned}
$$

What remains to be shown is to calculate the action of $Y^{S, R_{2}} z_{S}\left(e_{\alpha}\right)\left(Y^{S, R}\right)^{*}$. Let $\psi \in$ $\mathcal{F}_{S}(\mathcal{H})$ then

$$
\begin{aligned}
Y_{n+1}^{S, R} z_{S}^{*}\left(e_{\alpha}\right)\left(Y_{n}^{S, R}\right)^{*} \psi_{n} & =Y_{n+1}^{S, R} P_{n+1}^{S} e_{\alpha} \otimes\left(\left[Y_{1}^{S, R}\right]^{*}\right)^{\otimes n} \psi_{n} \\
& =P_{n+1}^{R}\left(Y_{1}^{S, R}\right)^{\otimes(n+1)}\left(e_{\alpha} \otimes\left(\left[Y_{1}^{S, R}\right]^{*}\right)^{\otimes n} \psi_{n}\right) \\
& =P_{n+1}^{R}\left(Y_{1}^{S, R} e_{\alpha}\right) \otimes \psi_{n} \\
& =z_{R}^{*}\left(Y_{1}^{S, R} e_{\alpha}\right) \psi_{n} .
\end{aligned}
$$

Thus the adjoint action of $Y^{\tilde{S}}, \tilde{R}$ results in an isomorphism between elements of the polynomial algebras $\mathcal{P}_{\tilde{S}}, \mathcal{P}_{\tilde{R}}$.

It must be mentioned, however, that the isomorphism of the Hilbert spaces given by $Y^{\tilde{S}, \tilde{R}}$ does not always give an isomorphism of Zamolodchikov representations. As a counter example, we restrict to dimension two and consider $S=-F_{\mathcal{H}} \sim-1 \boxplus-1=$ $R$. In the Fock representation of $\mathcal{Z}(S, \mathcal{L})$, we have anti-commutation between all annihilation operators, i.e.

$$
\left\{z_{\tilde{S}, \alpha}(f), z_{\tilde{S}, \beta}(g)\right\}=0
$$

for $\alpha, \beta \in\{1,2\}$ and all $f, g \in \mathcal{L}$. If an isomorphism between the representations of $\mathcal{Z}(S, \mathcal{L})$ and $\mathcal{Z}(R, \mathcal{L})$ existed, this anti-commutation would be preserved; however, for some choices of $\alpha$ and $\beta$, we actually have commutation in the representation of $\mathcal{Z}(R, \mathcal{L})$

$$
\left[z_{\tilde{R}, 1}(f), z_{\tilde{R}, 2}(g)\right]=0
$$


for all $f, g \in \mathcal{L}$. A quick calculation shows the product $z_{\tilde{R}, 1}(f) z_{\tilde{R}, 2}(g)$ is not zero, and thus, the representations $\pi_{\tilde{S}}, \pi_{\tilde{R}}$ are not isomorphic in this case.

Open Access This article is licensed under a Creative Commons Attribution 4.0 International License, which permits use, sharing, adaptation, distribution and reproduction in any medium or format, as long as you give appropriate credit to the original author(s) and the source, provide a link to the Creative Commons licence, and indicate if changes were made. The images or other third party material in this article are included in the article's Creative Commons licence, unless indicated otherwise in a credit line to the material. If material is not included in the article's Creative Commons licence and your intended use is not permitted by statutory regulation or exceeds the permitted use, you will need to obtain permission directly from the copyright holder. To view a copy of this licence, visit http://creativecommons.org/licenses/by/4.0/.

\section{References}

1. Abdalla, E., Abdalla, M., Rothe, K.: Non-perturbative Methods in Two-Dimensional Quantum Field Theory. World Scientific, Singapore (2001)

2. Bostelmann, H., Lechner, G., Morsella, G.: Scaling limits of integrable quantum field theories. Rev. Math. Phys. 23, 1115 (2011). https://doi.org/10.1142/S0129055X11004539

3. Bratteli, O., Robinson, D.W.: Operator Algebras and Quantum Statistical Mechanics 2. Springer, Berlin (1979)

4. Baez, J., Segal, I., Zhou, Z.: Introduction to Algebraic and Constructive Quantum Field Theory. Princeton University Press, Princeton (1992)

5. Castro-Alvaredo, O., Fring, A.: From integrability to conductance, impurity systems. Nucl. Phys. B 649, 449-490 (2003). arxiv: hep-th/0205076

6. Dubois-Violette, M., Landi, G.: Noncommutative products of Euclidean spaces. Lett. Math. Phys. 108(11), 2491-2513 (2018). https://doi.org/10.1007/s11005-018-1090-z. arxiv: 1706.06930

7. Evans, D., Kawahigashi, Y.: Quantum Symmetries on Operator Algebras. Oxford Science, Oxford (1998)

8. Faddeev, L.D.: Quantum completely integrable models of field theory. Sov. Sci. Rev. C 1, 107-155 (1980)

9. Fateev, V.A., Zamolodchikov, A.B.: Conformal field theory and purely elastic S matrices. Int. J. Mod. Phys. A 5, 1025-1048 (1990). https://doi.org/10.1142/S0217751X90000477

10. Haag, R.: Local Quantum Physics-Fields, Particles, Algebras, 2nd edn. Springer, Berlin (1996)

11. Jorgensen, P.E.T., Schmitt, L.M., Werner, R.F.: Positive representations of general commutation relations allowing Wick ordering. J. Funct. Anal. 134(1), 33-99 (1995). https://doi.org/10.1006/jfan.1995. 1139

12. Kuzmin, A., Ostrovskyi, V., Proskurin, D., Yakymiv, R.: On $q$-tensor product of Cuntz algebras. Preprint (2018). arxiv: 1812.08530

13. Lechner, G.: Polarization free quantum fields and interaction. Lett. Math. Phys. 64, 137-154 (2003). https://doi.org/10.1023/A:1025772304804. arxiv: hep-th/0303062

14. Lechner, G.: Algebraic constructive quantum field theory: Integrable models and deformation techniques. In: Advances in Algebraic Quantum Field Theory, pp. 397-448. Springer (2015). https://doi. org/10.1007/978-3-319-21353. arxiv: 1503.03822

15. Liguori, A., Mintchev, M.: Fock representations of quantum fields with generalized statistics. Commun. Math. Phys. 169, 635-652 (1995). arxiv: hep-th/9403039

16. Lechner, G., Pennig, U., Wood, S.: Yang-Baxter representations of the infinite symmetric group. Adv. Math., p. 106769(2019). https://doi.org/10.1016/j.aim.2019.106769. arxiv: 1707.00196v1

17. Lechner, G., Schützenhofer, C.: Towards an operator-algebraic construction of integrable global gauge theories. Ann. Henri Poincaré 15, 645-678 (2014). https://doi.org/10.1007/s00023-013-0260-x. arxiv: 1208.2366

18. Lukyanov, S.: Free field representation for massive integrable models. Commun. Math. Phys. 167(1), 183-226 (1995). https://doi.org/10.1007/BF02099357

19. Mishchenko, A.V.: On the braided Fock spaces. Ukr. Fiz. Zh. 41, 338-344 (1996). arxiv: hep-th/9701192 
20. Murgan, R., Nepomechie, R.: $q$-deformed $s u(2 \mid 2)$ boundary $S$-matrices via the ZF algebra. J. High Energy Phys. 6, 2008 (2008). https://doi.org/10.1088/1126-6708/2008/06/096

21. Pennig, U.: Exponential functors, R-matrices and twists. Preprint 4 (2018). arxiv: 1804.05807

22. Sviratcheva, K., Bahri, C., Georgieva, A., Draayer, J.: Physical significance of $q$-deformation and many-body interactions in nuclei. Phys. Rev. Lett. 93, 152501 (2004). https://doi.org/10.1103/ PHYSREVLETT.93.152501

23. Scotford, C.: Work in Progress; Ph.D. thesis

24. Smirnov, F.A.: Form Factors in Completely Integrable Models of Quantum Field Theory. World Scientific, Singapore (1992)

25. Zamolodchikov, A., Zamolodchikov, A.: Factorized $S$-matrices in two dimensions as the exact solutions of certain relativistic quantum field theory models. Ann. Phys. 120, 8 (1979). https://doi.org/10.1016/ 0003-4916(79)90391-9

Publisher's Note Springer Nature remains neutral with regard to jurisdictional claims in published maps and institutional affiliations. 